\title{
Covid-19: concerns grow over inflammatory syndrome emerging in children
}

\author{
Elisabeth Mahase
}

The BMJ

Doctors in the UK have been warned over a rising number of children presenting with a multisystem inflammatory state and needing intensive care.

In an urgent alert shared by North Central London Clinical Commissioning Group and the Paediatric Intensive Care Society, ${ }^{1}$ doctors were told that while the unspecified number of cases may be connected to the current pandemic, the symptoms have been observed in both children who have tested positive and negative for covid-19.

"There is a growing concern that a SARS-CoV-2 related inflammatory syndrome is emerging in children in the UK or that there may be another as yet unidentified infectious pathogen associated with these cases," the letter said.

Child health leaders have stressed that there is currently a small number of cases and that advice to parents regarding covid-19 has not changed.

The alert, which relayed information from NHS England, said, "It has been reported that over the past three weeks there has been an apparent rise in the number of children of all ages presenting with a multisystem inflammatory state requiring intensive care across London and other regions of the UK."

It noted that the cases have in common "overlapping features of toxic shock syndrome and atypical Kawasaki disease with blood parameters consistent with severe covid-19 in children.” Abdominal pain and gastrointestinal symptoms were also reported as common features, as well as cardiac inflammation.

"Please do refer patients as a matter of urgency if you encounter any children presenting with these types of symptoms," doctors have been told.

Adilia Warris, paediatric infectious diseases specialist at the University of Exeter, said, "Multisystem inflammatory state is a clinical presentation where a lot of cytokines are produced which dearrange a number of body functions, but the most important being leading to leaky blood vessels, causing low blood pressure and fluid accumulation in the lungs and other organs being in urgent need of intensive care treatment to support the function of the heart and the lungs, and sometimes other organs like the kidneys."

She cautioned, "Please do consider that the absolute number of those cases are very low, a handful at the moment. The call to ask if other colleagues have comparable experiences over the past week is so we are able to define what is going on."

Meanwhile Simon Kenny, NHS national clinical director for children and young people, told The BMJ that "it's important that clinicians are made aware of any potential emerging links so that they are able to give children and young people the right care fast."

But he added that the advice to parents remains the same. "If you are worried about your child for whatever reason, contact NHS 111 or your family doctor for urgent advice, or 999 in an emergency, and if a professional tells you to go to hospital, please go to hospital."

This message was echoed by Royal College of Paediatrics and Child Health president Russell Viner. "Parents should be reassured that children are unlikely to be seriously ill with covid-19 but if they are concerned about their children's health for any reason, they should seek help from a health professional," he said.

Increasing covid-19 cases in children have also raised concerns in the US. Speaking to the Washington Post, ${ }^{2}$ Roberta DeBiasi, infectious disease division chief at the Children's National Hospital in Washington, said, "We have been ready, but frankly surprised. We are actually having a significant number of admissions and critical care admissions."

Between 15 March and 22 April the hospitals saw 105 children who were sick with covid-19, 28 of whom required hospitalisation.

"It's nothing like the burden on elderly adults, but it's clear that it's not slowing down. We want people to be aware that it's true children aren't as heavily affected as elderly adults, but it does happen, and you need to be prepared, because it's not a rare thing either," said DeBiasi.

PICSUK 26 April 2020 https://twitter.com/PICSociety/status/1254508725227982848. 2 Swenson K. New DC hospital numbers suggest kids do face some risk of coronavirus hospitalization. 26 April 2020. Washington Post. www.washingtonpost.com/local/new-dchospital-numbers-suggest-kids-do-face-some-risk-of-coronavirus-hospitalization/2020/ 04/25/5e78c268-86fe-11ea-878a-86477a724bdb_story.html.

Published by the BMJ Publishing Group Limited. For permission to use (where not already granted under a licence) please go to http://group.bmj.com/group/rights-licensing/ permissions 